\title{
Overlapping Signal Separation in DPX Spectrum Based on EM Algorithm
}

\author{
Chuandang Liu" a , Luxi Lü, b \\ ${ }^{1}$ National Key Laboratory of Science and Technology on Blind Signal Processing. \\ aliuchuandang0603@126.com, byjluluxi@gmail.com
}

Keywords: Gamma Mixture Model, EM Algorithm, DPX.

\begin{abstract}
Overlapping signal separation in spectrum is a difficult problem. We use Gamma Mixture Model to formulate the distribution of signal power in each frequency bin of digital phosphor technology (DPX) spectrum. Then Expectation Maximization (EM) Algorithm is used to solve the model. Simulation results show that when CIR is greater than $2.7 \mathrm{~dB}$, parameters' estimation error rate of this algorithm is less than $1 \mathrm{e}-5$.
\end{abstract}

\section{Introduction}

The blind signal processing studies how to discover and analyze unknown, unlicensed and malicious signals. It attracts great interests of the spectrum supervision departments around the world. To exploit spectrum efficiently, supervision departments usually divide spectrum into pieces and authorize organizations or individuals to use some specified pieces in specified time and specified places. Thus, supervision departments will receive spectral overlapping signals. Before judging which signal is illegal, they need to separate these overlapping signals. However, it is difficult for traditional methods to separate overlapping signals because traditional methods like spectrum method and time-frequency method mentioned in [1] contain lots of averaging and smoothing calculation. These calculations will decrease the power response of burst signals in spectrum and even regard little signals as noise.

Digital Phosphor Technology (DPX) can store the power response during a period of time and display in a pseudo-color way [2]. Compared with traditional methods, DPX only counts the appearance time of signal power. Thus DPX spectrum contains undistorted power information of signals.

In this paper, we use Gamma Mixture Model to formulate the distribution of signal power in each frequency bin of DPX spectrum. Expectation Maximization (EM) Algorithm [3] is then adopted to calculate the model parameters which denote the power and the appearance time of signals.

\section{Analysis of Received Signals in DPX Spectrum}

Power Distribution of Received Signals in DPX. DPX counts time-frequency-power information obtained by Short-Time Fourier Transform (STFT) and compresses it into a 2-D histogram whose columns represent the trace of amplitude values and rows for points on the frequency axis. Each grid in the 2-D histogram stores the appearance number of times of signal whose power and frequency correspond to the column and row, respectively [4]. DPX is able to emphasize the signal of interests by pseudo-coloring method. However, there is no automatic method to recognize overlapping signals and separate them in DPX.

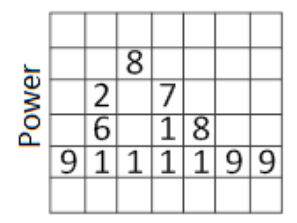

Frequency

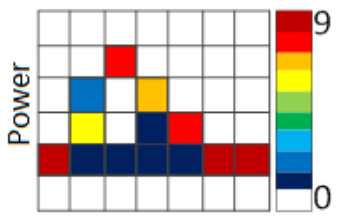

Frequency

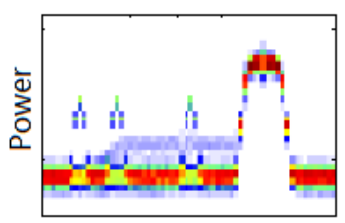

Frequency

Fig. 1 DPX Histogram, DPX Display Illustration and DPX Image of Signal 
Assume the received sampled signal is

$$
r(n)=\sum_{i=1}^{s} s_{i}(n)+v(n), n=0,1,2, \cdots
$$

where, $s_{i}(n)$ denotes the $\mathrm{i}$-th signal wave and $n$ is the sample index. Each signal can be narrow-band bursts, wide-band bursts or continued signal. $v(n)$ is noise and is usually not white in the broadband spectrum.

After the Discrete Fourier Transform (DFT) as (2), the spectrum is divided into multiple narrowband frequency bins.

$$
H\left(t, \omega_{f}\right)=\sum_{m=0}^{M-1} r(t M+m) e^{-i \omega_{f} m}=\sum_{i=1}^{S} H_{s_{i}}\left(t, \omega_{f}\right)+H_{v}\left(t, \omega_{f}\right),(f=0,1, \cdots, M-1 ; t=0,1,2, \cdots)
$$

where, $\omega_{f}=2 \pi f / M, f=0,1, \cdots, M-1$ is the frequency index, $M$ is the length of DFT and $t$ is the block index of DFT.

Let the bandwidth of each frequency bin be smaller than the channel correlation bandwidth. Therefore, $H_{v}\left(t, \omega_{f}\right)$ in each bin $\omega_{f}$ follows the complex Gaussian distribution with zero-mean and $\sigma_{f}^{2}$-variance (the noise power). The measurements of our approach are the average power in frequency $\omega_{f}$.

$$
R\left(t_{r}, \omega_{f}\right)=\frac{1}{N_{m}} \sum_{t=t_{r} N_{m}}^{t_{r} N_{m}+N_{m}-1}\left|H\left(t, \omega_{f}\right)\right|^{2}
$$

where $t_{r}=0,1,2, \cdots$ is the time index of measurements, $N_{m}$ is the average length. When $H\left(t, \omega_{f}\right)$ is noise only during one measurement time, according to the feature of complex Gaussian distribution, $R\left(t_{r}, \omega_{f}\right)$ follows Gamma distribution. The probability density function (PDF) of $R\left(t_{r}, \omega_{f}\right)$ is

$$
\phi\left(x \mid \lambda_{f}, N_{\mathrm{m}}\right)=\left\{\begin{array}{l}
\frac{\lambda_{f}}{\Gamma\left(N_{\mathrm{m}}\right)}\left(\lambda_{f} x\right)^{N_{\mathrm{m}}-1} \exp \left(-\lambda_{f} x\right), x>0 \\
0, x \leq 0
\end{array}\right.
$$

where, $\lambda_{f}=N_{\mathrm{m}} / \sigma_{f}^{2}$ contains the power information of signals or noise, and $\Gamma(\bullet)$ is Gamma Function.

Symbols in digital modulated signal approximately have independent uniform distribution. According to Central Limit Theorem, the greater $N_{m}$ is, the closer signal's power sum distribution approaches to Gaussian distribution, and the closer $R\left(t_{r}, \omega_{f}\right)$ approaches to Gamma distribution.

Actually, received signals in every frequency bin are the combination of pure signals and noise, and noise in one frequency bin can be regarded as an independent wideband signal. For simplicity, we use "signal" to express "received signal" or "noise".

Gamma Mixture Model in DPX Frequency Bin. There may be several signals in one frequency bin and the distribution of every signal's power follows Gamma Distribution. So we define a Gamma Mixture Model to formulate the power distribution in each frequency bin.

$$
P(\mathbf{y} \mid \boldsymbol{\theta})=\sum_{k=1}^{K} \alpha_{k} \phi\left(\mathbf{y} \mid \lambda_{k}\right)
$$

where, $K$ denotes the number of different Gamma distributions (equals to the number of noise and signals $). \phi\left(\mathbf{y} \mid \lambda_{k}\right)$ is the PDF of Gamma Distribution with parameter $\lambda_{k} \cdot \mathbf{y}=\left(y_{1}, y_{2}, \cdots, y_{N}\right)$ is an observation set of signal power in frequency bin $\omega_{f}, \boldsymbol{\theta}=\left(\alpha_{1}, \alpha_{2}, \cdots, \alpha_{K} ; \lambda_{1}, \lambda_{2}, \cdots, \lambda_{K}\right)$ is parameter vector, and $\alpha_{k}$ is the proportion of $\phi\left(\mathbf{y} \mid \lambda_{k}\right)$ and equals to the ratio that appearance number of times of $\phi\left(\mathbf{y} \mid \lambda_{k}\right)$ divide the total appearance number of times in frequency bin, subject to

$$
\sum_{k=1}^{K} \alpha_{k}=1
$$

Our work in this paper is to calculate vector $\boldsymbol{\theta}$ in each frequency bin. 


\section{EM Algorithm Solution of Gamma Mixture Model}

EM Algorithm was proposed by Dempster et al. in 1977. It is an iterative method to solve the Maximum Likelihood Estimation (MLE) problem of probability model parameters with hidden variables. It is proved in [3] and [5] that the iterative sequence of EM Algorithm converges to the stable point of logarithmic likelihood function sequence. Gamma Mixture Model in (5) fulfills the conditions to use EM Algorithm.

Let $\gamma_{j k}$ be the hidden variables of observation $y_{j}$,

$$
\gamma_{j k}=\left\{\begin{array}{l}
1, \text { if } y_{j} \text { is from } \phi\left(x \mid \lambda_{k}\right) \\
0, \text { otherwise }
\end{array}\right.
$$

The complete hidden variable vector to generate observation $y_{j}$ is $\gamma=\left(\gamma_{j 1}, \gamma_{j 2}, \cdots, \gamma_{j K}\right)$, whose likelihood function is

$$
P(\mathbf{y}, \boldsymbol{\gamma} \mid \boldsymbol{\theta})=\prod_{k=1}^{K} \alpha_{k}^{n_{k}} \prod_{j=1}^{N}\left[\phi\left(y_{j} \mid \lambda_{k}\right)\right]^{\gamma_{j k}}=\prod_{k=1}^{K} \alpha_{k}^{n_{k}} \prod_{j=1}^{N}\left[\frac{\lambda_{k}^{N_{m}} y_{j}^{N_{\mathrm{m}}-1} \exp \left(-\lambda_{k} y_{j}\right)}{\Gamma\left(N_{\mathrm{m}}\right)}\right]^{\gamma_{j k}}
$$

where $N=\sum_{k=1}^{K} n_{k}, n_{k}=\sum_{j=1}^{N} \gamma_{j k}$.

Derive the Q-function of $P(\mathbf{y}, \boldsymbol{\gamma} \mid \boldsymbol{\theta})$

$$
Q\left(\boldsymbol{\theta}, \boldsymbol{\theta}^{(i)}\right)=\sum_{k=1}^{K}\left\{\sum_{j=1}^{N}\left(E \gamma_{j k}\right) \log \alpha_{k}+\sum_{j=1}^{N}\left(E \gamma_{j k}\right)\left[-\log \Gamma\left(N_{\mathrm{m}}\right)+N_{\mathrm{m}} \log \lambda_{\mathrm{k}}+\left(N_{\mathrm{m}}-1\right) y_{j}-\lambda_{\mathrm{k}} y_{j}\right]\right\}
$$

where, $i$ denotes the iteration index, and $E \gamma_{j k}$ denotes the expectation of hidden variable $\gamma_{j k}$.

Then we use Bayes Formula to compute $E \gamma_{j k}$ in (9).

$$
E \gamma_{j k}=\frac{P\left(y \mid \gamma_{j k}=1, \boldsymbol{\theta}\right) P\left(\gamma_{j k}=1 \mid \boldsymbol{\theta}\right)}{\sum_{k=1}^{K} P\left(y \mid \gamma_{j k}=1, \boldsymbol{\theta}\right) P\left(\gamma_{j k}=1 \mid \boldsymbol{\theta}\right)}=\frac{\alpha_{k}\left[\phi\left(y_{j} \mid \lambda_{k}\right) \Delta_{k}\right]}{\sum_{k=1}^{K} \alpha_{k}\left[\phi\left(y_{j} \mid \lambda_{k}\right) \Delta_{k}\right]},\left\{\begin{array}{l}
j=1,2, \cdots, N \\
k=1,2, \cdots, K
\end{array}\right.
$$

where, $\Delta_{k}$ is the integration interval width of $\phi\left(x \mid \lambda_{k}\right)$ in logarithmic horizontal coordinate.

Last, we need to derive the MLE of Q-function. Let the partial derivatives of (9) with respect to $\lambda_{k}$ and $\alpha_{k}$ equal to 0 (subject to (6)), thus we have

$$
\hat{\lambda}_{\mathrm{k}}=\left(N_{\mathrm{m}} \sum_{j=1}^{N} \hat{\gamma}_{j k}\right) /\left(\sum_{j=1}^{N} \hat{\gamma}_{j k} y_{j}\right), \hat{\alpha}_{k}=\left(\sum_{j=1}^{N} \hat{\gamma}_{j k}\right) / N
$$

We can get a sufficient good solution of $\boldsymbol{\theta}$ by iterating (10) and (11) until stopping conditions fulfilled. Stopping criterion for iteration can be a difference threshold of parameter vector $\boldsymbol{\theta}$ between adjacent iterations.

\section{Processing Flow of EM Algorithm}

Potential signal amount (parameter $K$ in (5)) in each frequency bin of DPX spectrum is an important input of EM algorithm. We find that the proportions of existing signals are approximately the real value and the spare proportions are approximately zero, if $K$ is greater than the real number of signals.

Table 1 shows the calculation results of one frequency bin in Fig. 5 with an increasing $K$. Actually there are two signals in the frequency bin in total. When $K$ is greater than 2, spare proportions are approximately equal to 0 .

Table 2 0shows the EM Algorithm results of some frequency bin in Fig. 5 when $K=5 . k$ denotes the signal number of Gamma Distribution $\phi$. All $\alpha_{k}$ are between 0.1 and 0.3 , but the values of $\sigma_{k}^{2}$ are all around $-66.74 \mathrm{dBm}$ and vary less than $1 \%$. Thus, these five results should be regarded as one signal. In fact, there is only one signal in that frequency bin. 
Table 1 Proportion Calculation Results of Some Frequency Bin in Fig. 5

\begin{tabular}{ccccc}
$K$ & $\alpha_{1}$ & $\alpha_{2}$ & $\alpha_{3}$ & $\alpha_{4}$ \\
\hline 1 & 1.0000 & - & - & - \\
2 & 0.7177 & 0.2823 & - & - \\
3 & 0.0000 & 0.7177 & 0.2823 & - \\
4 & 0.0000 & 0.0000 & 0.7177 & 0.2823 \\
\hline
\end{tabular}

Table 2 EM Algorithm Results of Some Frequency Bin in Fig. 5, $K=5$

\begin{tabular}{ccc}
$k$ & $\alpha_{k}$ & $\sigma_{k}^{2}(\mathrm{dBm})$ \\
\hline 1 & 0.1416 & -66.734 \\
2 & 0.1670 & -66.737 \\
3 & 0.1960 & -66.741 \\
4 & 0.2290 & -66.744 \\
5 & 0.2663 & -66.747 \\
\hline
\end{tabular}

Then we propose a selection strategy of $K$ as follows: 1) set a threshold to filter the results with small signal proportions; 2) quantify signal power and merge the signals with close power; 3 ) process all frequency bins with step 1) and step 2) and mark the results with pseudo-color. The entire processing flow is shown in Fig. 2.

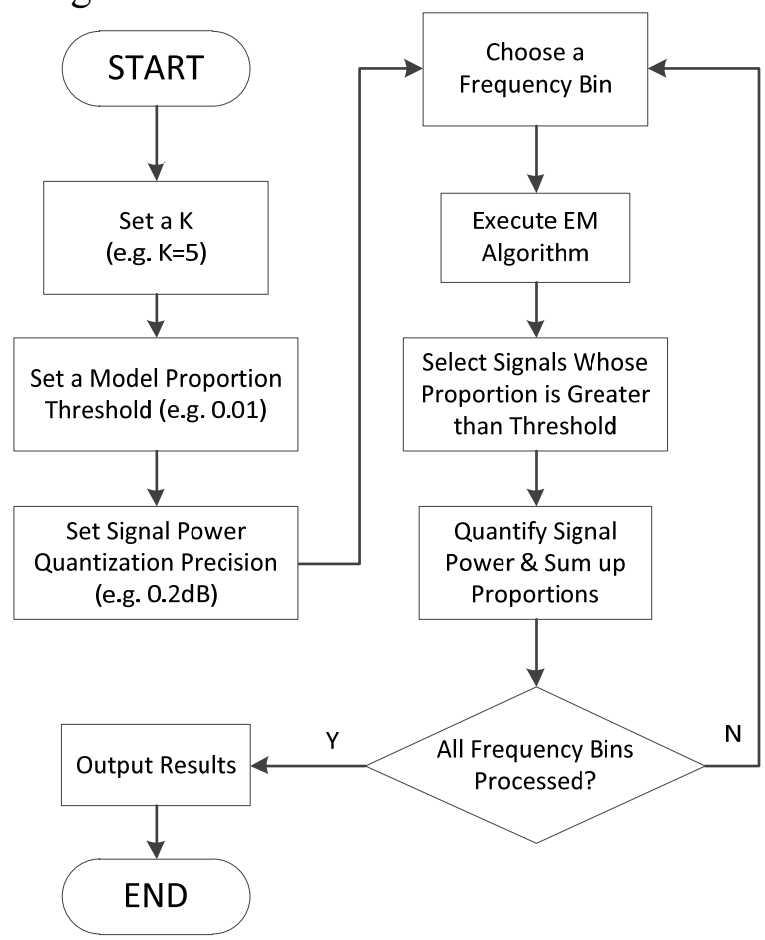

Fig. 2 Parameters Estimation Algorithm Flow

\section{Algorithm Performance Analysis and Simulation}

We use Monte-Carlo Simulation to calculate signal separation error rate. To avoid the occasional error, we record the number of Monte-Carlo simulation times when signal separation error occurs 100 times. In every Monte-Carlo simulation we generate 1000 power samples in a frequency bin. The error criterion of signal separation is defined that the difference between calculated power value and the real power value is greater than $0.25 \mathrm{~dB}$ or calculated proportion differs from the real ones more than 0.05 .

Let $N_{m}=40, \alpha_{N}=0.5$ and CIR increase from $0.5 \mathrm{~dB}$ to $4 \mathrm{~dB}$. Simulation results in Fig. 3 shows that the error rate decreases along with CIR. When CIR is greater than $2.7 \mathrm{~dB}$, error rate is less than 1e-5. The reason is that the distances between signals' distribution maximums depend on CIR. The higher the CIR is, the more distinct the signal is from the interference. 
Let $\alpha_{N}=0.5, \mathrm{CIR}=3 \mathrm{~dB}, 4 \mathrm{~dB}, 5 \mathrm{~dB}, 6 \mathrm{~dB}$, and $N_{m}$ increase from 2 to 40 . Simulation results in Fig. 4 demonstrates that the error rate decreases along with $N_{m}$. According to the Central Limit Theorem, signal power distribution approaches closer to Gamma Distribution along with the increase of $N_{m}$, so the estimation will be more accurate when $N_{m}$ is higher.

Fig. 5 shows the DPX image of simulation signal. The length of DFT in DPX is 512. Simulation signals contain: 1) one $-67 \mathrm{dBm}$ continued BPSK signal with $26.25 \mathrm{MHz}$ carrier frequency and $4.4 \mathrm{MHz}$ bandwidth; 2) one $-79 \mathrm{dBm}$ burst BPSK signal with $16.3 \mathrm{MHz}$ carrier frequency and $8.75 \mathrm{MHz}$ bandwidth; 3 ) a set of frequency hopping BPSK signals whose carrier frequency set is $\{5.5$, $8.2,10.9,13.6,16.3\} \mathrm{MHz}$, bandwidth of each hop is $1 \mathrm{MHz}$ and power is $-72 \mathrm{dBm}$; and 4) one $8.1 \mathrm{dBm}$ complex white Gaussian noise. Simulation signals' separation result is showed in Fig. 6.

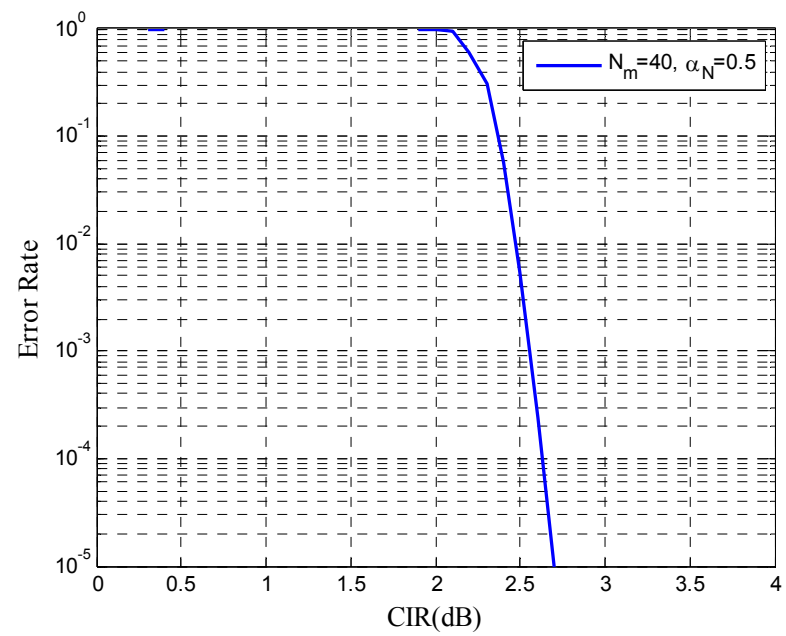

Fig. 3 Parameters Estimation Error Rate Curve

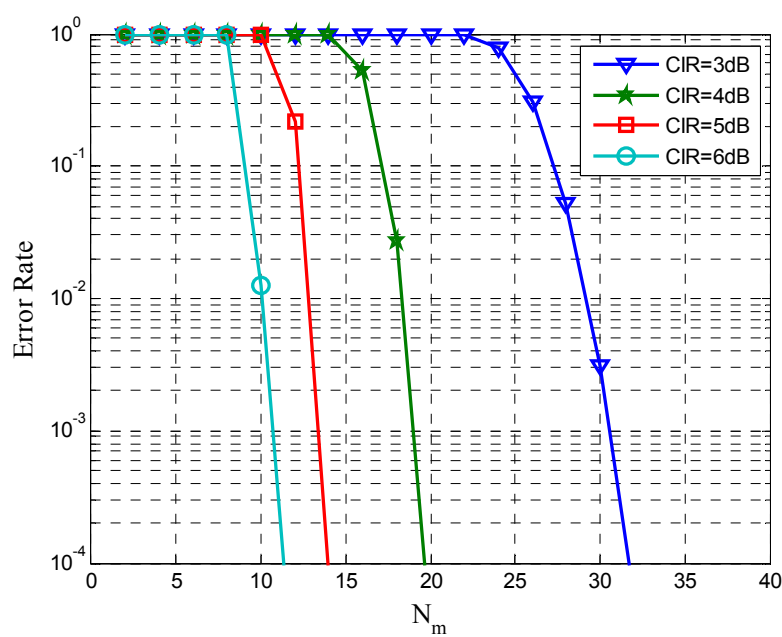

Fig. 4 Parameters Estimation Error Rate Curve 


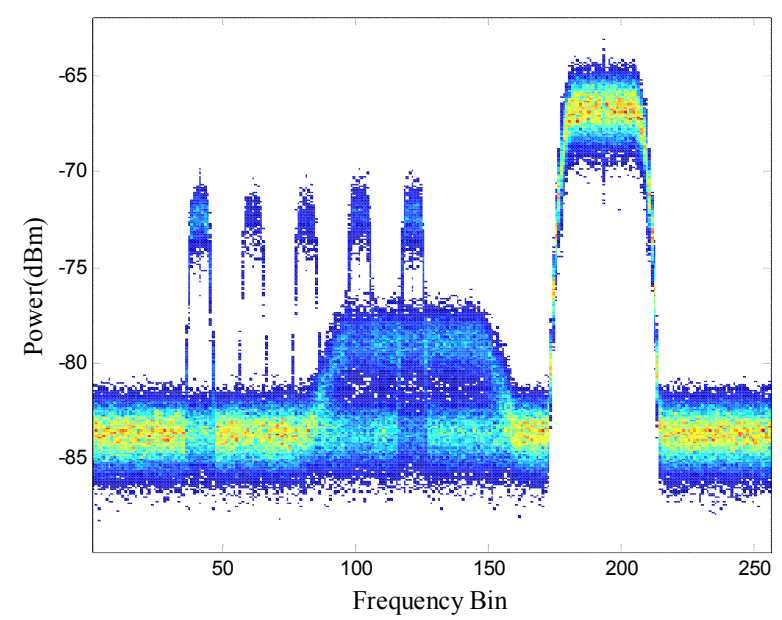

Fig. 5 DPX Image of Simulation Signal

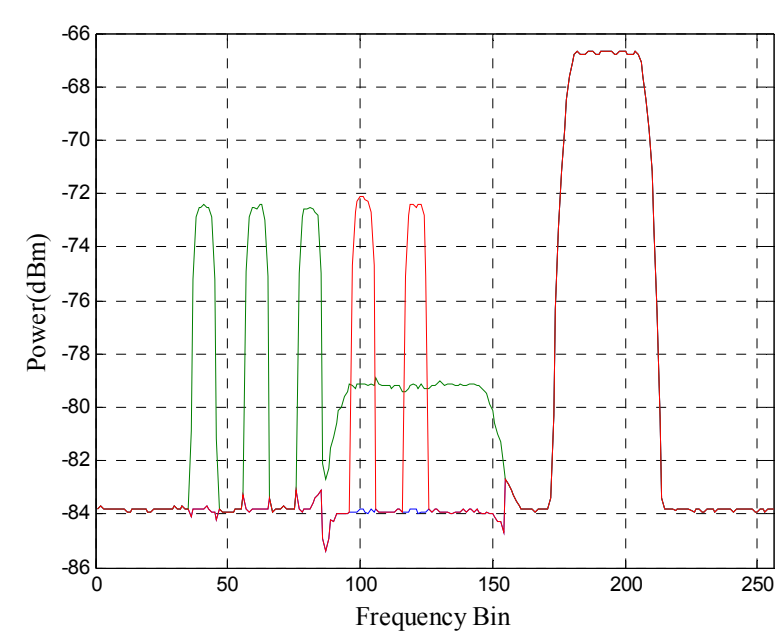

Fig. 6 Simulation Signal Classification and Visualization

\section{Conclusion}

In this paper, we focus on the overlapping signal separation. We use Gamma Mixture Model to formulate the power distribution of mixed signals in each frequency bin of DPX spectrum. Then EM Algorithm is adopted to solve this model. Our simulation results demonstrate that the error rate of the algorithm is less than $1 \mathrm{e}-5$ when CIR is greater than $2.7 \mathrm{~dB}$.

Further work will be directed to a precise signal classification and recognition.

\section{References}

[1] Tektronix, Fundamentals of Real-Time Spectrum Analysis. (2009)

[2] Tektronix, DPX Acquisition Technology for Spectrum Analyzers Fundamentals Primer. (2009)

[3] P. Dempster, N. M. Laird and D. B. Rubin, Maximum Likelihood from Incomplete Data via E-M Algorithm, Journal of the Royal Statistical Society, 39 (1977).

[4] S. Guo, J. Su, G. Sun, Z. Zhao, and Z. Chen, Design of DPX System for Real-Time Spectrum Analysis and Signal Detection, IEICE Electronics Express, 10 (2013) 1-8.

[5] G. McLachlan and T. Krishnan, The EM Algorithm and Extensions, New York: John Wiley \& Sons, 1996. 